\title{
Short sequence repeats (SSR) under selection pressure: Cyprinidae fish case study
}

\author{
Mikhail Orlov \\ ICB RAS, Pushchino, Russia \\ orlovmikhailanat@gmail.com
}

\begin{abstract}
Short sequence repeats (SSR) were earlier shown to have length well correlated with various genomic hotspots including intense selection pressure, chromosome rearrangement, etc. We approach this by considering SSR sets for numerous Cyprinidae fish representatives. Analysis of length of SSR as well as their oligonucleotide composition revealed difference between domesticated vs free-living fish.
\end{abstract}

Keywords - SSR, short tandem repeats, Cyprinidae, genetics, selection

Motivation and aim

Motivation

Genes encoding traits under strict selection pressure were shown to have SSR (short tandem repeats) with length well correlated with the quantity of the traits [2]. SSR are capable of rapid mutational changes known as expansions (i.e. trinucleotide expansions) which are connected to the rate of various evolutionary processes [3].

Aim

Cyprinidae representatives are characterized by complex polyploidy, interspecies and interphylum hybridization as well as massive chromosome rearrangement events [1]. Here we describe SSR for 28 Cyprinidae fish (including particularly interesting and important goldfish Carassius auratus).

\author{
Andrey Tykhonov \\ "Aqua Logo" company group, Moscow, Russia \\ andrew693@mail.ru
}

\section{Methods}

SSR data were taken from FishMicrosat database [4]. For each set corresponding to a species we have considered lengths and nucleotide composition. Namely, di-, tri- and tetranucleotide frequency was derived. The result was furtherly clusterized using hierarchical Ward's method.

\section{Results}

Accordly to the hypothesis we put forward, domesticated fish clustered mostly together. So did the free-living species. The same oligo frequency dataset was analysed using principal component analysis ( $\mathrm{pcA}$ ). This reinforced the result above while also showing significant difference between the two clusters. Indeed, TG/CA dinucleotide presented more in domesticated fish with smaller amount of AG/TC dinucleotide. This mifht suggest the presence of specific SSR region with some relation to genes under evolutionary pressure.

\section{REFERENCES}

[1] Fondon J. W., Garner H. R. // 2004, V. 101(52), P. 18058-18063

[2] K. T. Xie, G. Wang, A. C. Thompson et al. // Science. 2019. V. 363. P. 81-84.

[3] Katsnelson L.B. et al. Boron A., Spoz A., Porycka K. et al. Comparative Cytogenetics. 2014, V. 8(3), P.233-248.

[4] Nagpure N.S., Rashid I., Pati R. et al. BMC Genomics. 2013, V. 14, P. 630. 